\title{
El hospital en los tiempos del Covid-19: capacidad de reacción y adaptación
}

\author{
Hospital in times of Covid-19: reaction and adaptation
}

\author{
Jesús Conde-Freire ${ }^{1}$, Beatriz García-Trincado ${ }^{2}$, David Rubal-Bran² ${ }^{2}$ Pablo Ventura-Valcárcel ${ }^{2}$, Héctor Guerrero-Sande ${ }^{2}$, \\ Elena Rodríguez-Ameijeiras², Manuel Francisco Liroa-Romero², María Matesanz-Fernández², Iria Íñiguez-Vázquez², \\ Emilio Casariego-Vales ${ }^{2}$ \\ ${ }^{1}$ Servicio de Documentación Clínica. Hospital Universitario Lucus Augusti. Lugo. ${ }^{2}$ Servicio de Medicina Interna. Hospital Universitario Lucus Augusti. Lugo
}

\section{RESUMEN}

Objetivo: Describir las repercusiones sobre la hospitalización y las características de los pacientes atendidos en las primeras semanas tras la declaración del estado de alarma durante la pandemia por COVID-19 en un hospital general.

Métodos: Estudio observacional de todos los ingresos, en todos servicios hospitalarios, entre los días 1 de marzo y 30 de abril de los años 2017, 2018, 2019 y 2020 en un hospital general. La fuente de información fue el conjunto mínimo básico de datos del centro. Consideramos las 00.00 horas del día 14 de marzo como el inicio del estado de alarma y punto de corte entre dos periodos: previo al estado de alarma (días 1 a 13 de marzo) y estado de alarma (días 14 a 30 de abril).

Resultados: Tras la declaración del estado de alarma disminuyó el número de hospitalizaciones ( $p<0.0001$ ), en un rango entre el $3,5 \%$ y el $55,9 \%$ con respecto al promedio de los 3 años previos en los diez principales servicios médicos y quirúrgicos de adultos y por todas las modalidades de ingreso $(p<0.001)$. En paralelo se redujo la estancia media $(p<0.001)$ y se incrementó el porcentaje de ingresados de procedencia urbana $(p<$ 0.01). Si bien la mortalidad global no mostró cambios, si aumentaron los fallecidos en las primeras 24 horas de ingreso hospitalario ( $p<0.008$ ).

Conclusiones: Este estudio describe los mecanismos de reacción y adaptación de un hospital durante el estado de alarma por la pandemia por COVID-19. Nuestros resultados podrían ayudar a otros centros a diseñar y dimensionar sus preparativos.

Palabras clave: COVID-19 Pandemia, Capacidad de reacción, gestión hospitalaria.

\section{INTRODUCCIÓN}

La pandemia causada por el coronavirus 2019 (COVID-19) ha impactado sobre los sistemas de salud, el bienestar social y la economía a nivel mundial en una proporción que no tiene parangón en la historia moderna ${ }^{1,2}$. Además, el crecimiento exponencial de la enfermedad ${ }^{3}$ ha obligado a adoptar medidas asistenciales y sociales no habituales, tanto en España como en otros países europeos. Por una parte, ha sido preciso focalizar múltiples recursos sanitarios en la asistencia de estos pacientes, especialmente a aquellos con enfermedad crítica. Por otra, el decreto de estado de alarma ha modificado la vida cotidiana de todos los ciudadanos, como no había sucedido en las últimas décadas, y provocado profundos cambios sociales y económicos ${ }^{4-6}$.

A nivel hospitalario, además de los efectos de la enfermedad por si misma, se generan nuevos problemas colaterales. Entre ellos se incluyen el sobre aforo $0^{7,8} 0$ el retraso, cuando

\begin{abstract}
Aim: Describe the patient's features and the hospital changes during the first weeks of the COVID-19 pandemic alarm in a General Hospital.

Method: Observational study that asses all the admissions in the hospital departments between March $1^{\text {st }}$ and April 30 $30^{\text {th }}$ of 2017, 2018, 2019 and 2020 in a General Hospital. The information was obtained from the basic data set of the Center. We consider 00.00 on March $14 \mathrm{t}^{\mathrm{h}}$ of 2020 as the beginning of the alarm state and as cut-off point between two periods: before the state of alert (March $1^{\text {st }}-13^{\text {th }}$ ) and the state of alert (March $14^{\text {th }}$ - April $30^{\text {th }}$ )

Results: After the state of alarm the number of admissions decreased $(p<0,0001)$ in all kind of admissions $(p<0,001)$ and in the ten medical and surgical services of adults between $3,5 \%$ and $55,9 \%$ comparing with the main of 3 previous years. At the same time main stay decreased $(p<0,001)$ and rate of admitted from urban areas increased $(p<0,01)$. Although total mortality did not change, deaths during the first 24 hours after admissions were increased $(p<0,008)$.

Conclusions: This study describes surge and adaptation mechanisms of a hospital during state of alert by COVID-19 pandemic. Our results could help other Centers with designing and measuring their preparations.
\end{abstract}

Keywords: COVID-19 pandemic, Reaction capacity, Hospital Management

no la cancelación, de múltiples consultas, pruebas o intervenciones ${ }^{9,10}$. Si bien se han estudiado las necesidades y los costes que requirieron otras pandemias como influenza ${ }^{11,12}$, los efectos y daños, secundarios o adicionales, provocados por el COVID-19 son aún poco conocidos ${ }^{13}$. Por ejemplo, poco se sabe sobre cual es la demanda asistencial en una situación de estado de alarma o la repercusión que tiene sobre la asistencia hospitalaria general ${ }^{14}$. Este conocimiento ha de ser clave para establecer las medidas que permitan resolver esta situación con eficacia y equidad.

Por ello, analizamos las repercusiones sobre la hospitalización y las características de los pacientes atendidos en las primeras semanas de la declaración del estado de alarma en un hospital general que da servicio a una población con una tasa de incidencia media por COVID1915. 


\section{PACIENTES Y MÉTODOS}

Estudio observacional de todos los ingresos en todos servicios hospitalarios entre los días 1 de marzo y 30 de abril de los años 2017,2018,2019 y 2020 en el Hospital Universitario Lucus Augusti de Lugo. Este centro cubre la asistencia sanitaria de 240.000 habitantes y dispone de 879 camas.

La fuente de información fue el conjunto mínimo básico de datos del centro. Las variables analizadas fueron: sexo, edad, servicio de ingreso, tipo de ingreso, destino al alta, estancia hospitalaria, código postal y municipio de residencia. Todos los pacientes fueron seguidos hasta su alta o fallecimiento durante el mismo ingreso hospitalario. Se decidió considerar las 00.00 horas del día 14 de marzo como el inicio del estado de alarma puesto que se anunció horas antes de su entrada en vigor y se entendió que ese era el momento en que comenzó a tener relevancia a los efectos de este estudio. Este momento es el punto de corte entre dos periodos: pre-estado de alarma (días 1 a 13 de marzo) y estado de alarma ( días 14 a 30 de abril). La variable tipo de ingreso se distribuyó en las siguientes categorías: Urgente procedente de Urgencias, urgente procedente de Consultas Externas, programado, traslado desde otro Centro y otros. Se catalogó como zona urbana el área centro del municipio de Lugo y como zona rural el resto de la provincia. Los datos se incluyen en un registro aprobado por el Comité Ético de Investigación del Centro y en todos los casos la información recogida fue anonimizada. No se accedió a ninguna historia clínica concreta, respetando la normativa vigente sobre la confidencialidad de los datos.

En el análisis estadístico se utilizaron las técnicas descriptivas habituales. En los cálculos de porcentajes se utilizó como referencia el número de ingresos en todos los casos. Se usó el test ji al cuadrado para la comparación de variables cualitativas, con la corrección de Yates cuando fue preciso. Para la comparación de 2 medias se utilizó, previa comprobación de la homocedasticidad, el test t de Student. En la comparación múltiple de medias se utilizó el análisis de la varianza y el test de Duncan. El nivel de significación estadística se estableció en $p<0,05$. Se utilizó el paquete estadístico SPSS versión 17.0.

\section{RESULTADOS}

Entre el 1 de marzo y el 30 abril de los años 2017, 2018, 2019 y 2020 ingresaron un total de 13481 pacientes, 13237 de ellos de 14 o más años. Entre estos últimos 7007 (52.9\%) fueron varones. La edad media fue 69.74 (DS 18,28) años, sin que se apreciasen diferencias por sexos. Las características generales de las hospitalizaciones de estos pacientes se muestran en la Tabla 1. En este periodo de tiempo de 2020, comparado con el mismo periodo de 2017, 2018 y 2019, el número de hospitalizaciones se redujo de manera muy evidente $(p<0.0001)$. Simultáneamente algunas de las características de los pacientes, como el incremento de casos de procedencia urbana $(p<0.01)$, se modificaron en 2020 con respecto a años previos. Por otra parte, también cambiaron algunas características de los ingresos, como la
Tabla 1. Características generales de los pacientes ingresados en el hospital de Lugo entre el 1 de marzo y el 30 de abril de 2017, 2018, 2019 y 2020

\begin{tabular}{|c|c|c|}
\hline & $\begin{array}{l}\text { TOTAL INGRESOS } \\
\quad(n=13237)\end{array}$ & $p$ \\
\hline Sexo; varón ( n; \%) & 7007 ( 52,9\%) & \\
\hline $\begin{array}{c}\text { Edad ( media; DS) } \\
\text { Global } \\
\text { Varones } \\
\text { Mujeres }\end{array}$ & $\begin{array}{l}69,74(18,28) \\
69,51(16,89) \\
69,99(16,67)\end{array}$ & ns \\
\hline $\begin{array}{l}\left.\text { Ingresos por año ( } \mathrm{n}^{0} ; \%\right) \\
2017 \\
2018 \\
2019 \\
2020\end{array}$ & $\begin{array}{l}3377(25,5) \\
3645(27,5) \\
3601(27,2) \\
2614(19,7)\end{array}$ & $<0,0001$ \\
\hline $\begin{array}{c}\text { Tipo de ingreso } \\
\text { Urgente desde Urgencias } \\
\text { Programado } \\
\text { Urgente/ Consultas } \\
\text { Traslado a otro centro } \\
\text { Otros }\end{array}$ & $\begin{array}{l}8330(62,9) \\
2759(20,8) \\
1115(8,4) \\
211(1,6) \\
822(6,3)\end{array}$ & \\
\hline $\begin{array}{c}\text { Lugar de residencia (urbano/ } \\
\text { total/\%) } \\
2017 \\
2018 \\
2019 \\
2020\end{array}$ & $\begin{array}{c}1193 / 2183 / 35,3 \\
1189 / 2380 / 33,3 \\
1212 / 2349 / 24,0 \\
906 / 1542 / 37,1\end{array}$ & $<0,01$ \\
\hline $\begin{array}{l}\text { Fallecidos totales ( } \mathrm{n} \% \text { total) } \\
\qquad \begin{array}{c}2017 \\
2018 \\
2019 \\
2020\end{array}\end{array}$ & $\begin{array}{l}258 / 3119 \\
307 / 3338 \\
282 / 3319 \\
237 / 2377\end{array}$ & ns \\
\hline $\begin{array}{c}\text { Fallecidos en las } 1^{\circ} 24 \mathrm{~h} \\
\text { (fallecidos } 1^{\circ} 24 \mathrm{~h} / \text { total) } \\
2017 \\
2018 \\
2019 \\
2020\end{array}$ & $\begin{array}{l}49 / 258 \\
45 / 307 \\
51 / 282 \\
69 / 237\end{array}$ & 0,0001 \\
\hline
\end{tabular}

menor estancia media $(p<0.0001)$ o la mayor mortalidad en las primeras 24 horas del ingreso $(p<0.0001)$.

En las Tabla 2 se muestran diferentes aspectos de los pacientes y sus hospitalizaciones en dos periodos de tiempo: preestado de alarma (días 1 a 13 de marzo) y estado de alarma (14 de marzo a 30 de abril). Para cada variable en estudio se muestran los datos de los 4 años consecutivos en estudio. Destaca que, coincidiendo con el periodo de alarma de 2020, se incrementan el porcentaje de ingresados de procedencia urbana $(p<0.01)$, disminuyen de manera homogénea todos las modalidades de ingreso $(p<0.0001)$, se redujo la estancia media $(p<0.001)$ y se incrementan los fallecidos en las primeras 24 horas de ingreso hospitalario $(p<0.008)$. Por el contrario no se apreciaron diferencias ni en la distribución por sexos, en la edad de los ingresados ni en la mortalidad global. Durante el estado de alarma se redujo, en los diez servicios médicos y quirúrgicos con mayor tasa de hospitalizaciones, 
Tabla 2. Características de los pacientes ingresados en el hospital de Lugo en los periodos de tiempo coincidentes con el pre-estado y estado de alarma de 2017,2018,2019 y 2020

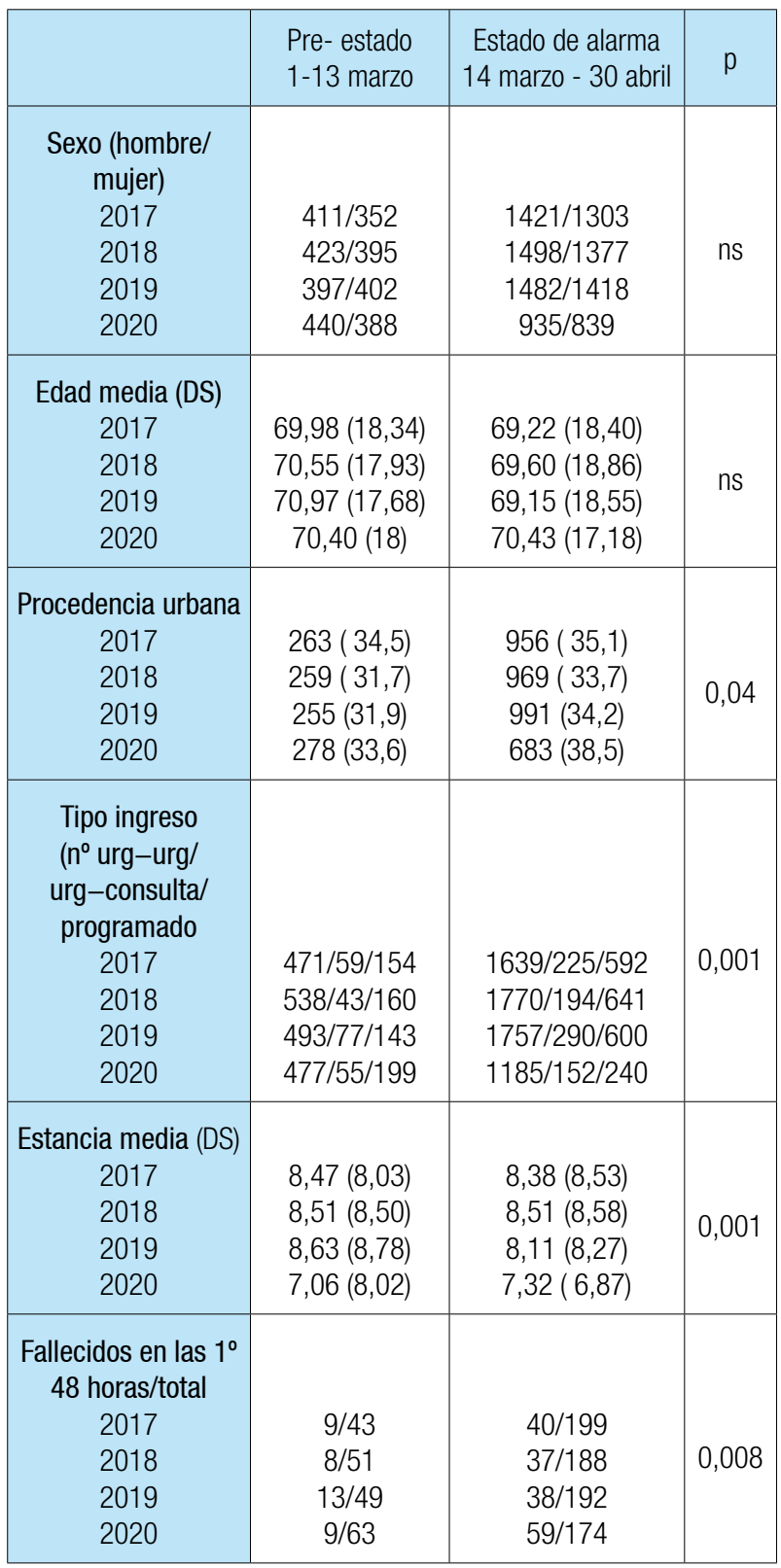

el número de ingresos de manera muy llamativa (Tabla 3). Sin embargo la disminución fue muy distinta según los servicios. La asociación de los Servicios de Neumología y Medicina Interna (con enfermedades infecciosas), que se encargó conjuntamente de los enfermos con COVID-19 durante este periodo, atendió un número de pacientes levemente inferior al de años precedentes. De la misma manera el número de pacientes atendidos por Hospitalización a Domicilio se redujo un 3,8\%. Sin embargo los restantes Servicios disminuyeron su actividad en hospitalización entre el 33\% de UCl y el 55,9\% de Geriatría.

En el periodo de tiempo que abarca nuestro artículo en el área de referencia del Centro se diagnosticaron, con PCR positiva,
Tabla 3. Diferencias en la ocupación durante en los años 2017,2018 y 2019 en el mismo periodo del estado de alarma de 2020 para diferentes servicios.

\begin{tabular}{|c|c|c|c|c|c|}
\hline \multicolumn{7}{|c|}{ INGRESOS ESTADO ALARMA (13/03-30/04) } \\
\hline & 2017 & 2018 & 2019 & 2020 & $\begin{array}{c}\text { Variación } \\
\%\end{array}$ \\
\hline $\begin{array}{c}\text { MEDICINA } \\
\text { INTERNA }+ \\
\text { NEUMOLOGÍA }\end{array}$ & 724 & 735 & 761 & 636 & $-6,8 \%$ \\
\hline CIRUGÍA & 302 & 284 & 313 & 139 & $-53,6 \%$ \\
\hline TRAUMATOLOGÍA & 218 & 224 & 219 & 105 & $-52,3 \%$ \\
\hline GERIATRÍA & 170 & 209 & 213 & 87 & $-55,9 \%$ \\
\hline DIGESTIVO & 119 & 154 & 147 & 75 & $-46,2 \%$ \\
\hline UROLOGÍA & 127 & 140 & 162 & 89 & $-37,7 \%$ \\
\hline CARDIOLOGÍA & 141 & 145 & 156 & 72 & $-51,1 \%$ \\
\hline $\begin{array}{c}\text { HOSPITALIZACIÓN } \\
\text { A DOMICILIO }\end{array}$ & 122 & 97 & 120 & 109 & $-3,5 \%$ \\
\hline $\begin{array}{c}\text { UNIDAD CUIDADOS } \\
\text { INTENSIVOS }\end{array}$ & 94 & 130 & 121 & 77 & $-33 \%$ \\
\hline GINECOLOGÍA & 125 & 111 & 100 & 70 & $-37,5 \%$ \\
\hline
\end{tabular}

un total de 746 pacientes. En el pico de la pandemia el número máximo de ingresados simultáneos fue de 69 pacientes en hospitalización y 21 en la Unidad de Cuidados Intensivos. Fallecieron 18 pacientes.

\section{DISCUSIÓN}

Este estudio muestra como en el hospital de un área con una influencia media por COVID-19 durante el estado de alarma se redujeron las hospitalizaciones en todos los servicios y el tiempo de estancia, ingresó un mayor porcentaje de pacientes de procedencia urbana y, aunque no se incrementó la mortalidad global, si lo hicieron los fallecidos en las primeras 24 horas.

Los hospitales han de estar, en todo momento, totalmente operativos para dar una respuesta adecuada a todo tipo de desastres, como una pandemia. Capacidad de reacción (surge capacity) es el término usado para describir la competencia de un hospital para asumir una sobrecarga repentina de pacientes por un motivo concreto y sin dejar de atender otras patologías urgentes ${ }^{16}$. Para ello las acciones estratégicas más habituales son cancelar intervenciones, reducir los ingresos programados no urgentes y acelerar, en lo posible, las altas. Se considera que con todo ello es posible reducir la ocupación en un $34 \%$ en el plazo de una semana ${ }^{14}$. En nuestro caso esta estrategia permitió reducir todos los tipos de ingreso y la ocupación disminuyo en más de un $50 \%$ en 4 de los 10 servicios principales (Tablas 1,2 y 3). Entendemos que este efecto fue muy superior a lo esperado y que se justifica por la suma de varios factores: una tasa de incidencia media por COVID-19 en nuestra área, un manejo intra-hospitalario adecuado de los 
pacientes que permitió que la ocupación máxima no superase el $10 \%$ de las camas del hospital ${ }^{17}$, una preparación previa muy exitosa y una baja afluencia de pacientes durante el estado de alarma. Una de las características de las pandemias víricas es que actúan de manera repentina y con un impacto variable en distintas zonas, hasta el punto de que no es posible predecir cuales serán las más afectadas. Por ello la preparación ha de efectuarse en todos los hospitales y diseñada para que no sean desbordados, incluso en las circunstancias más adversas $^{18}$. Sin embargo, la baja ocupación que registramos no puede atribuirse solamente a una tasa de incidencia media 0 a una preparación adecuada. Un factor determinante en nuestro caso ha sido la baja afluencia de pacientes durante el estado de alarma; un fenómeno que todavía ha de ser estudiado en su justa medida ${ }^{13}$. En todo caso no siempre es posible descifrar o explicar numéricamente los sentimientos que están detrás de las decisiones que toman las personas. Y no sólo ha de valorarse la capacidad de reacción hospitalaria. Nuestros datos sugieren que tanto pacientes como personal sanitario intentan adaptase a la nueva situación. Por parte de los pacientes, acudir al hospital durante una crisis como la del COVID-19 podría generar inseguridad 0 que minimizasen sus síntomas. Esto podría explicar la menor afluencia a Urgencias y la reducción de los ingresos en todos los servicios, independientemente de su relación con el COVID-19 (Tabla 3). Además, el incremento leve de la edad y del porcentaje con residencia más cercana al hospital pueden interpretarse como formas de autoselección. Es decir, los pacientes intentan adaptarse a la situación y evitan, en la medida de los posible, el ingreso hospitalario. Por parte de los profesiones es llamativa la reducción de hasta un día de la estancia media en este periodo. Entendemos que es una adaptación, probablemente inconsciente, que pretende bajar la presión sobre el hospital ante el temor de un incremento de los ingresos. Más difícil es explicar el leve, pero significativo, aumento de la mortalidad en las primeras 24 horas de ingreso sin que se modifique la mortalidad total. Este deslizamiento temporal puede tener interpretaciones muy diversas. Una opción sería que los casos de mayor gravedad acudan al hospital en etapas más evolucionadas de su enfermedad. Otra opción, no descartable, es que en esta situación de alarma un mayor número de pacientes y familias decidan que un fallecimiento se produzca en el hospital ${ }^{18}$.

Este estudio debe valorarse teniendo en cuenta sus limitaciones. En primer lugar son datos de un único hospital y no necesariamente similares a otros Centros de nuestro entorno. Si bien esto es cierto, nuestro estudio puede ser útil para diseñar nuevos y más amplios estudios que ayuden a preparar el control de crisis futuras. Una segunda cuestión es que este estudio analiza la capacidad de respuesta de un hospital al inicio de la pandemia y no es útil más allá de los dos primeros meses de crisis. En tercer lugar, en nuestra área sanitaria, las tasas de incidencia de la pandemia fueron intermedias entre las registradas en las distintas Comunidades Autónomas. Por tanto nuestras cifras pudiesen no ser extrapolables a lugares con tasas de incidencia mucho más altas o bajas.

En resumen, este estudio contribuye al análisis de los mecanismos de reacción y adaptación de un hospital durante el estado de alarma por la pandemia por COVID-19. Nuestros resultados podrían ayudar a otros centros a diseñar y dimensionar sus preparativos. Sin embargo, son necesarios nuevos estudios que confirmen y amplíen estos resultados en otras áreas geográficas.

\section{BIBLIOGRAFÍA}

1. WHO. WHO Virtual press conference on COVID-19. March 11, 2020. Disponible en: https://www.who.int/docs/default-source/coronaviruse/transcripts/whoaudio-emergencies-coronavirus-press-conference-full-and-final-11mar2020. pdf?sfvrsn=cb432bb3_2 . Consultado el 30 de abril de 2020 .

2. Instituto Nacional de Estadística. Información estadística para el análisis del impacto de la crisis COVID-19. Disponible en: https://www.ine.es/covid/covid_ inicio.ht . Consultado el 28 de mayo de 2020.

3. Ministerio de Sanidad. Situación de COVID-19 en España. Disponible en https:// covid19.isciii.es/. Consultado 24 mayo de 2020.

4. KPMG. Gestionar el impacto del COVID-19. Claves para que las empresas gestionen y minimicen los impactos del COVID-19. Disponible en: https://home. kpmg/es/es/home/tendencias/2020/03/gestionar-impacto-covid-19.html Consultado el 24 de mayo de 2020

5. FUNCAS. COVID-19: impacto económico y social. Disponible en:https://www. funcas.es/covid-19/. Consultado el 24 de mayo de 2020

6. European AntiPoverty Network. COVID-19: Impacto en personas vulnerables. Disponible en: https://www.eapn.es/covid19/impacto. Consultado el 24 de mayo de 2020

7. Orús A. COVID-19: provincias con mayor sobrecarga hospitalaria España 2020. https://es.statista.com/estadisticas/1104286/provincias-con-mayor-sobrecargahospitalaria-por-covid-19-espana/\#statisticContainer

8. Nekoie-Moghadam M, Kurland L, Moosazadeh M, Ingrassia PL, Della Corte F, Djalali A. Tools and Checklists Used for the Evaluation of Hospital Disaster Preparedness: A Systematic Review. Disaster Med Public Health Prep. 2016 ;10(5):781-788.

9. Verheul ML, Dückers M, Visser BB, Beerens RJ, Bierens J. Disaster Exercises to Prepare Hospitals for Mass-Casualty Incidents: Does It Contribute to Preparedness or Is It Ritualism?. Prehosp Disaster Med. 2018;33(4):387-393.

10. Kain T, Fowler R. Preparing Intensive Care for the Next Pandemic Influenza. Crit Care. 2019;23(1):337.

11. Lau K, Hauck K, Miraldo M. Excess influenza hospital admissions and costs due to the 2009 H1N1 pandemic in England. Health Economics. 2019; 28: 175-188.

12. Daugherty Biddison EL, Faden R, Gwon HS, Mareiniss DP, Regenberg AC, SchochSpana M, Schwartz J, Toner ES. Too Many Patients... A Framework to Guide Statewide Allocation of Scarce Mechanical Ventilation During Disasters. Chest. $2019 ; 155(4): 848-854$.

13. Conde-Freire J , Pérez López A, Rodríguez-Álvarez A, Gil-Moure C , RodríguezAmeijeiras E, Liroa-Romera MF et al. Pandemia por covid19: otras consecuencias. Galicia Clin (2021; 82-1: 5-8).

14. Jen HC, Shew SB, Atkinson JB, Rosenthal JT, Hiatt JR. Creation of Inpatient Capacity During a Major Hospital Relocation: Lessons for Disaster Planning. Arch Surg. 2009;144(9):859-64.

15. Ministerio de Sanidad. Centro de Coordinación de Alertas y Emergencias Sanitaria. Actualización nº 68. Enfermedad por el coronavirus (COVID-19). https://www. mscbs.gob.es/profesionales/saludPublica/ccayes/alertasActual/nCov-China/ documentos/Actualizacion_68_COVID-19.pdf. Consultado el 10 de mayo de 2020.

16. Davis DP, Poste JC, Hicks T, Polk D, Rymer TE, Jacoby I. Hospital Bed Surge Capacity in the Event of a Mass-Casualty Incident. Prehosp Disaster Med. 2005; 20(3):169-76

17. García-Trincado B , Conde Freire J , Liroa-Romero MF, Ferreiro-González A, Rodríguez-Ameijeiras E, Casariego-Vales E. . El hospital en los tiempos del COVID-19: ingresos y mortalidad. Galicia Clin, (en prensa).

18. Jiménez-Puente A, García-Alegría J. Distribución geográfica y evolución de las muertes en hospitales en España, 1996-2015. Rev Clin Esp 2018; 218: 285-292. 